\title{
Field-insensitive heavy fermion features and phase transition in the caged-structure quasi-skutterudite $\mathrm{Sm}_{3} \mathrm{Ru}_{4} \mathrm{Ge}_{13}$
}

\author{
Harikrishnan S. Nair ${ }^{1 *}$, Ramesh Kumar K. ${ }^{1}$, Douglas Britz ${ }^{1}$, Sarit K. Ghosh ${ }^{1,2}$, Christian Reinke ${ }^{3}$, André M. \\ Strydom ${ }^{1,4,5 *}$ \\ ${ }^{1}$ Highly Correlated Matter Research Group, Physics Department, P. O. Box 524, University of Johannesburg, Auckland Park 2006, South Africa \\ ${ }^{2}$ Department of Applied Physics, Birla Institute of Technology, Mesra-835215, Ranchi, Jharkhand, India \\ ${ }^{3}$ Analytical Facility, P. O. Box 524, University of Johannesburg, Auckland Park 2006, South Africa. \\ ${ }^{4}$ Institute of Physics, Chinese Academy of Sciences, PO Box 603, Beijing 100190, China \\ ${ }^{5}$ Max Planck Institute for Chemical Physics of Solids (MPICPfS), Nöthnitzerstraße 40, 01187 Dresden, Germany
}

\begin{abstract}
The robust field-insensitive heavy fermion features in $\mathrm{Sm}_{3} \mathrm{Ru}_{4} \mathrm{Ge}_{13}$ and the magnetic phase transition at $T_{N} \approx 5 \mathrm{~K}$ are studied using magnetization $M(T)$, specific heat $C_{p}(T)$, resistivity $\rho(T)$ and thermal conductivity $\kappa_{T}(T)$. The average crystal structure of $\mathrm{Sm}_{3} \mathrm{Ru}_{4} \mathrm{Ge}_{13}$ conforms to the cubic space group $\operatorname{Pm} \overline{3} n$ however, signatures of subtle structural distortions are obtained from the $\mathrm{x}$ ray data. The magnetic susceptibility, $\chi(T)$, follows a modified Curie-Weiss law indicating the presence of crystal fields of $\mathrm{Sm}^{3+}$ and the significance of van Vleck terms. No sign of ferromagnetism is observed in $M(H)$ of $\mathrm{Sm}_{3} \mathrm{Ru}_{4} \mathrm{Ge}_{13}$ which yields only $0.025 \mu_{\mathrm{B}} / \mathrm{f}$.u.-Sm at $2 \mathrm{~K}, 7 \mathrm{~T}$. The Sommerfeld coefficient, $\gamma \approx 220 \mathrm{~mJ} / \mathrm{mol}-\mathrm{Sm} \mathrm{K} \mathrm{K}^{2}$, estimated from the analysis of low temperature specific heat suggests the formation of heavy quasi particles at low temperature. Though a $\ln T$ dependence of $\rho(T)$ is observed till $60 \mathrm{~K}$, the resistivity behavior is accounted for by assuming a two-band model for activated behavior of charge carriers. The field scans of resistivity, $\rho(H)$, below $T_{N}$ display significant nonlinearity while those above the $T_{N}$ are more metal-like. Low values of thermal conductivity, $\kappa_{T}(T)$, are observed in $\mathrm{Sm}_{3} \mathrm{Ru}_{4} \mathrm{Ge}_{13}$ however, displaying an anomaly at $T_{N}$ which signifies magnetoelastic coupling. A fairly high value of Seebeck coefficient, $S \approx 40 \mu \mathrm{V} / \mathrm{K}$ is observed at $300 \mathrm{~K}$. We identify $\mathrm{Sm}_{3} \mathrm{Ru}_{4} \mathrm{Ge}_{13}$ as a low charge carrier density system with unusual field-insensitive heavy fermion features very similar to the filled skutterudites.
\end{abstract}

Keywords: Quasi-skutterudite, Field-insensitive, Samarium, Heavy fermion

\section{Introduction}

Intermetallics with caged structures stimulate deep interest in strongly correlated electron physics because of the possibility of heavy fermion physics, superconductivity, "rattling" of atoms and thermoelectricity [1, 2, 3, 4, 5, 5]. The caged structure are also known for field-insensitive heavy fermion state [7, 8] and mixed-valence [9, 10]. For example, the filled-skutterudites of chemical formula $R T_{4} X_{12}(R=\operatorname{rare}$ earth, $T=$ transition metal and $X=\mathrm{P}$, As or $\mathrm{Sb}$ ) display heavy fermion superconductivity, multipole ordering and unusual field-insensitive features in specific heat and in resistivity [11, 12, 7] which are also observed in the Frank-Kasper type caged structure intermetallics, $R T_{2} X_{20}$ [13, 14, 15]. Both $R T_{4} X_{12}$ and $R T_{2} X_{20}$ display puzzling physics especially when $R=\operatorname{Sm}[16,17,18,5,7,19]$. The field-insenstive phase transition in some of the Sm-based $R T_{2} X_{20}$ has been attributed to octupolar ordering[16]. Though Sm-based heavy fermion systems are limited (e.g., $\mathrm{SmPd}_{3}$ with $\gamma=280 \mathrm{~mJ} / \mathrm{mol} \mathrm{K}^{2}$ and $\mathrm{SmFe}_{4} \mathrm{P}_{12}$ with $\gamma=370 \mathrm{~mJ} / \mathrm{mol} \mathrm{K}{ }^{2}$ ), exotic heavy fermion state has been reported in the filled skutterudites $\mathrm{SmOs}_{4} \mathrm{Sb}_{12}$ [7] and

*h.nair.kris@gmail.com, hsnair@uj.ac.za 


\section{$\mathrm{SmRu}_{4} \mathrm{P}_{12}[17]$.}

The ternary intermetallic compounds denoted by the nominal stoichiometry $R_{3} T_{4} X_{13}$ [where $R$ is either a rareearth element, an early $d$-block element such as Sc or Y, or an alkali-earth metal like $\mathrm{Ca}$ or $\mathrm{Sr} ; T$ stands for a Group VIII $d$-electron element and $X$ is $\mathrm{In}, \mathrm{Ge}$, or $\mathrm{Sn}]$ with $R=\mathrm{Sm}$ are less-explored systems in connection with heavy fermion physics or field-insensitive phase transitions. The first report on $R_{3} T_{4} X_{13}$ phase is generally accredited to Remeika et al,[20]. The crystal structure of the $R_{3} T_{4} X_{13}$ series is identified by the cubic space group $P m \overline{3} n$ which is common to most of the compounds in this class, but the literature refers to the archetypal $R_{3} T_{4} X_{13}$ phase as either the $\mathrm{Pr}_{3} \mathrm{Rh}_{4} \mathrm{Sn}_{13}$-structure type [21], or the $\mathrm{Yb}_{3} \mathrm{Rh}_{4} \mathrm{Sn}_{13}$-type [22]. This structure type allows a single crystallographic site for occupation by each of the $R$ and $T$ atoms, and two distinct sites are available to the $X$ atom. Following the initial discovery, $R_{3} T_{4} X_{13}$ attracted attention mainly due to the discovery of superconductivity with superconducting temperatures as high as $8 \mathrm{~K}$ being reported in $\mathrm{Yb}_{3} \mathrm{Rh}_{4} \mathrm{Sn}_{13}$ and $\mathrm{Ca}_{3} \mathrm{Rh}_{4} \mathrm{Sn}_{13}$ [20], and has remained a topic of recent interest [23, 24]. In $\mathrm{Y}_{3} \mathrm{Ir}_{4} \mathrm{Ge}_{13}$, peculiar structural aspect of the formation of atomic cages was illustrated and its effect on thermal transport and other properties related to lattice vibration modes were elucidated[25]. Charge ordering has also been suggested in this class of materials, for example, in $\mathrm{Yb}_{3} \mathrm{Ir}_{4} \mathrm{Ge}_{13}$ [26].

A schematic of the crystal structure of $\mathrm{Sm}_{3} \mathrm{Ru}_{4} \mathrm{Ge}_{13}$ is shown in Fig 1 highlighting the caged structure motifs. In the figure, the big green spheres are Sm, the small black spheres are Ru and the gray spheres forming the polyhedra are the Ge atoms. Two kinds of cages - one centered at Ge ( $2 a$ position) and Sm ( $6 c$ position) - are shown as gray cages. The empty Ge-cage is not depicted in the figure. A clear understanding of subtle details of distortions in the crystal structure is essential to understand the physics of $R_{3} T_{4} X_{13}$. Remeika et al., first reported the crystal structure of $R_{3} T_{4} X_{13}$ in a cubic primitive unit cell however, also suggested plausible face-centered and tetragonal structures [20]. Hodeau et al., reported a body-centered unit cell for the same compound [27]. Modifications to the cubic structure with changes in stoichiometry were suggested[28]. The first two reports on the crystal structure of the germanides are from Segre et al., [29] and from Bruskov et al., [30] who proposed a disordered variant of the crystal structure where Ge atoms partially occupy two different $24 k$ sites. Positional disorder of the $X$ atom or the formation of random mixture of $R / T$ and $X$ atoms is also known [31, 32]. Doubling of the unit cell to a noncentrosymmetric $I 4_{1} 32$ space group has also been observed in some of the stannides of $R_{3} T_{4} X_{13}$ [33, 34]. In this paper we investigate a Sm-based quasi-skutterudite named $\mathrm{Sm}_{3} \mathrm{Ru}_{4} \mathrm{Ge}_{13}$ motivated to explore similar physics as has been observed in $R T_{4} X_{12}$ or $R T_{2} X_{20}$ concerning exotic heavy fermions states or the field-insensitive phase transitions. In fact, through our structural, magnetic, thermodynamic and transport investigations of the title compound we observe robust field-insensitive heavy fermion features.

\section{Experimental Details}

Polycrystalline samples of $\mathrm{Sm}_{3} \mathrm{Ru}_{4} \mathrm{Ge}_{13}$ were prepared by arc melting the constituent elements $\mathrm{Sm}(4 N), \mathrm{Ru}$ $(4 N)$ and $\mathrm{Ge}(4 N)$ together, according to stoichiometric ratio, in an Edmund Buehler arc melting furnace under static atmosphere of purified argon gas. A Zr-getter trap was used for purifying the Ar gas. The once-melted sample was re-melted 4 times in order to ensure a homogeneous single phase. The weight loss of the samples after arc melting was found to be not greater than $3 \%$. The melted samples, wrapped in Ta foils, were annealed in an evacuated quartz tube for 7 days at $900{ }^{\circ} \mathrm{C}$. Powder $\mathrm{X}$ ray diffractogram on pulverized sample of $\mathrm{Sm}_{3} \mathrm{Ru}_{4} \mathrm{Ge}_{13}$ was obtained using a Philips $\mathrm{X}$ 'pert diffractometer using $\mathrm{Cu} \mathrm{K} \alpha$ radiation. Structural analysis of the powder data was performed using Rietveld method[35] implemented in FullProf suite of programs [36]. The phase purity was probed using backscattered-electron imaging and X-ray microanalysis using a Cameca SX 100 electron microprobe (EPMA). The electron microprobe analysis was performed on optically polished samples, measuring the intensities of the $\mathrm{L} \alpha$ lines of the rare-earth elements, the $\mathrm{K} \alpha$ lines of the transition metals and the second-order $\mathrm{K} \alpha$ line of germanium with wavelength-dispersive spectrometers. The probe was calibrated on rare-earth orthophosphates [37, 38] and pure metals, respectively. The measured X-ray intensities were converted to weight fractions using the X-PHI matrix correction in Cameca's Peak Sight software (ver. 4.0) [39]. Magnetic measurements were performed using a commercial Magnetic Property Measurement System and specific heat was measured using a commercial Physical Property Measurement System (both instruments from M/s Quantum Design). The specific heat of a non-magnetic reference compound $\mathrm{Sm}_{3} \mathrm{Ru}_{4} \mathrm{Ge}_{13}$ was also measured. Electrical resistivity was measured on a bar-shaped sample using the ac transport option of the PPMS. A similar bar-shaped sample (roughly $7.5 \mathrm{~mm} \times 2 \mathrm{~mm} \times 1 \mathrm{~mm}$ ) was used for thermal conductivity 
measurements using the Thermal Transport Option of the PPMS which measures the thermal conductivity, electrical resistivity and Seebeck coefficient simultaneously.

\section{Results}

\subsection{Crystal structure}

Following the electron microprobe analysis (EPMA), the elemental concentration of the sample was ascertained as $\mathrm{Sm}_{3.54} \mathrm{Ru}_{3.91} \mathrm{Ge}_{11.85}$ which is in close proximity with the nominal composition but with a deficiency of Ge. The chemical composition is estimated as the average from 7 different positions on the sample surface. A photograph of the microstructure in two different magnifications are shown in Fig 2 (a) and (b). The darker phase seen in (b) corresponds to the binary $\mathrm{Ru}_{2} \mathrm{Ge}_{3}$ in orthorhombic Pbcn space group. As mentioned in Section 1 , the crystal structure of $R_{3} T_{4} X_{13}$ compounds is commonly described in the cubic space group $P m \overline{3} n$ (\#223). Recent crystallographic studies on $R_{3} T_{4} X_{13}$ [40] as well as early work[22] point towards subtle structural distortions and variants which may be present in these structure types. Rietveld refinement trials of the $x$ ray data on $S m_{3} \mathrm{Ru}_{4} \mathrm{Ge}_{13}$ was performed first using the space group $P m \overline{3} n$. Subsequently, additional phases of $\mathrm{Ru}_{2} \mathrm{Ge}_{3}(P b c n)$ was added in the refinement cycles. The results of the refinement are presented in Fig 3 where the experimentally observed data are shown in circles, the calculated pattern in thick solid line and the allowed Bragg positions as vertical tick marks. The Bragg peaks corresponding to the major phase of $\mathrm{Sm}_{3} \mathrm{Ru}_{4} \mathrm{Ge}_{13}(88 \%)$ and the minor phases $\mathrm{Ru}_{2} \mathrm{Ge}_{3}(12 \%)$ are differentiated using different colors in the figure. The magnetic behaviour of $\mathrm{Ru}_{2} \mathrm{Ge}_{3}$ is described as temperature-independent in the temperature range 50-900 K [41, 42]. We conclude that this binary phase which unintentionally co-melted with the major phase is of no consequence in the analysis and findings of the results obtained on the title compound $\mathrm{Sm}_{3} \mathrm{Ru}_{4} \mathrm{Ge}_{13}$. In the inset (1) of panel (a), the (400) reflection from the cubic unit cell of $\mathrm{Sm}_{3} \mathrm{Ru}_{4} \mathrm{Ge}_{13}$ is shown magnified. A comparison with the report of Gumeniuk et al., [40] shows that a possible tetragonal distortion that splits the cubic (400) reflection in to tetragonal (440) and (004) reflections may be present. The evolution of full-width-at-half-maximum (FWHM) with $2 \Theta$ is shown in the inset (2) where an abrupt change is observed close to $40^{\circ}$. These observations hint at possible structural distortions that could be present, however, requires detailed high resolution scattering data to confirm. The details of the structural model including the atomic coordinates, refined lattice parameters and goodness-of-fit are collected in Table 1. The lattice parameters of the binary phases are also given. The isotropic thermal parameters, $B_{\text {iso, }}$, were collected from Ref.[43] and the occupancies were fixed to nominal values. In order to ascertain whether other structural variants or distortions plausible for $R_{3} T_{4} X_{13}$ are present in $\mathrm{Sm}_{3} \mathrm{Ru}_{4} \mathrm{Ge}_{13}$, we performed Rietveld refinement trials using permitted space groups $P 4_{2} \mathrm{~cm}, P 4_{2} \mathrm{mcm}, I 2_{1} 3, \operatorname{Im} \overline{3}$ for this type of structure [40, 22]. The unit cell parameters, $a=b=12.7497, c=9.0214$ were used for $P 4_{2} \mathrm{~cm}$ and $P 4_{2} \mathrm{mcm} ; a=18.0578$ for $I 2_{1} 3$ and $I m \overline{3}$. It was noticed that the $P 4_{2} \mathrm{~cm}$ space group offered a reasonable fit to the observed data however, the intensities were not completely faithfully accounted for. Within the limited resolution of our laboratory $\mathrm{x}$ ray data, it was not possible to quantify the subtle structural distortions present in $\mathrm{Sm}_{3} \mathrm{Ru}_{4} \mathrm{Ge}_{13}$.

\subsection{Heat capacity}

The experimentally measured zero-field specific heat of $\mathrm{Sm}_{3} \mathrm{Ru}_{4} \mathrm{Ge}_{13}$ is presented in Fig 4 (a) as circles. A prominent $\lambda$-like transition is observed at $T_{N} \approx 5 \mathrm{~K}$. In the high-temperature region $T>300 \mathrm{~K}$, the Dulong-Petit value $3 n R \approx 498 \mathrm{~J} / \mathrm{mol} \mathrm{K}$ is recovered ( $n$ is the number of atoms in the unit cell and $R$ is the universal gas constant). Also shown in (a) is the specific heat of the non-magnetic analogue, $\mathrm{La}_{3} \mathrm{Ru}_{4} \mathrm{Ge}_{13}$ as a blue solid line. The inset of (a) shows the magnetic entropy $S_{\text {mag }}$ estimated after subtracting the specific heat of $\mathrm{La}_{3} \mathrm{Ru}_{4} \mathrm{Ge}_{13}$ from that of $\mathrm{Sm}_{3} \mathrm{Ru}_{4} \mathrm{Ge}_{13}$ The values of $S=R \ln (2 J+1)$ corresponding to $J=5 / 2$ and 7/2 are marked in the figure with dotted horizontal lines.

Figure 4 (b) shows the experimental $C_{p}(T)$ measured at applied fields of 1, 3 and 5 T. The zero-field $C_{p}$ is also re-plotted in this graph for the sake of completeness. It is remarkable to note that the specific heat is insensitive to changes in applied magnetic field; up to $5 \mathrm{~T}$ in the present case. Such field-insensitive behavior is observed in Sm-based caged structure systems like $\operatorname{SmTa}_{2} \mathrm{Al}_{20}$ and $\operatorname{SmTi}_{2} \mathrm{Al}_{20}$ [13, 16]. The robustness of $C_{p}(T)$ observed under magnetic field for $\mathrm{Sm}_{2} \mathrm{Ta}_{2} \mathrm{Al}_{20}$ was explained in the parlance of multipole moments in the quartet ground state of Sm ion. A collection of relevant thermodynamic parameters of some of the $R_{3} T_{4} \mathrm{Ge}_{13}$ are presented in Table 2 . It can be readily seen that $\mathrm{Sm}_{3} \mathrm{Ru}_{4} \mathrm{Ge}_{13}$ has a high $\gamma$ values among most of the Ge-based compounds. Fig 4 (c) shows the plot of $C_{p} / T$ versus $T^{2}$. The linear region immediately above the $T_{N}$ was fitted with the expression $C_{p}(T)=\gamma T+\beta T^{3}$ (shown 
in red solid line). The value of the Sommerfeld coefficient $\gamma$ estimated from the fit is $220 \mathrm{~mJ} / \mathrm{mol}-\mathrm{Sm} \mathrm{K}{ }^{2}$. A $\gamma$ value of $14 \mathrm{~mJ} / \mathrm{mol} \mathrm{K}^{2}$ was obtained for the Kondo system, $\mathrm{Yb}_{3} \mathrm{Os}_{4} \mathrm{Ge}_{13}$ [43]. A value of $280 \mathrm{~K}$ was extracted for $\Theta_{D}$ from this low temperature fit. For a comparison, the $\gamma$-values obtained for some of the Sm-containing intermetallics are: $\mathrm{SmV}_{2} \mathrm{Al}_{20}=720 \mathrm{~mJ} / \mathrm{mol} \mathrm{K}{ }^{2}$ and $\mathrm{SmCr}_{2} \mathrm{Al}_{20}=1000 \mathrm{~mJ} / \mathrm{mol} \mathrm{K}{ }^{2}$ [15]. A significantly high value of $\gamma$ for $\mathrm{Sm}_{3} \mathrm{Ru}_{4} \mathrm{Ge}_{13}$ clearly shows that the effective electron mass has been enhanced.

In Fig $4(\mathrm{~d})$, a plot of $\left(C_{p}(T)-\gamma T\right) / T^{3}$ versus $T$ is shown. In the quasi-skutterudite compounds with strong "rattling" modes, such a plot reveals the phonon contribution most clearly [25, 44]. In the case where strong phonon modes are present, the $\left(C_{p}(T)-\gamma T\right) / T^{3}$ versus $T$ or $C_{p}(T) / T^{3}$ versus $T$ plots would produce a clear anomaly at $T_{\max } \approx \Theta_{D} / 5[25]$. Germanide quasi-skutterudites, $\mathrm{Y}_{3} \mathrm{Ir}_{4} \mathrm{Ge}_{13}$ and 1: 2: 20 alloys like $\mathrm{LaV}_{2} \mathrm{Al}_{20}$ [15] are known to display such features. However, in the present case of $\mathrm{Sm}_{3} \mathrm{Ru}_{4} \mathrm{Ge}_{13}$, strong phononic anomalies suggestive of "rattling" are not evident. The low temperature specific heat in the magnetically ordered region below the $T_{N}$ was analyzed to test for the type of magnetic ordering. Antiferromagnets display $C_{p}(T) \sim T^{2}$ or $T^{2} \exp \left(-\Delta / k_{B} T\right)$ where $\Delta$ is the magnon gap. In the panel (e) of Fig 4 a curve-fit is attempted on the low temperature specific heat below $T_{N}$ using the above-mentioned equation. However, it was found that there exists two regions below $T_{N}$ with slightly differing temperature dependencies and using a modified expression, $T^{n} \exp \left(-\Delta / k_{B} T\right)$, it was found to be $n \approx 1.45$ and 2.20, repsectively. This suggests that the magnetic ordering in $\mathrm{Sm}_{3} \mathrm{Ru}_{4} \mathrm{Ge}_{13}$

\subsection{Magnetization}

The magnetic response of $\mathrm{Sm}_{3} \mathrm{Ru}_{4} \mathrm{Ge}_{13}$ is presented in Fig 5. The temperature evolution of magnetic susceptibility in the field-cooled mode of measurement applying $2 \mathrm{~T}$ magnetic field is shown in Fig 5 (a) as black circles. The magnetic phase transition which was observed in the specific heat at $T_{N} \approx 5 \mathrm{~K}$ is present in the magnetization curve as an anomaly. A conspicuous feature of the magnetic susceptibility, $\chi(T)$, of $\mathrm{Sm}_{3} \mathrm{Ru}_{4} \mathrm{Ge}_{13}$ is the nonlinearity present in the whole temperature range of measurement which is evident in the $1 / \chi(T)$ plot given in the inset of (a). The departure from Curie-Weiss behaviour may originate from the crystalline electric field (CEF) effects of the $\mathrm{Sm}^{3+}$, taking into account the proximity of the $J=\frac{5}{2}$ ground state and the first excited state with $J=\frac{7}{2}$. The high temperature magnetic susceptibility of Sm compounds can have significant contributions from van Vleck terms [45]. The fact that valence fluctuations and crystal field effect contributions play a role in the magnetic susceptibility of Sm-based intermetallics is shown in the case of $\mathrm{SmFe}_{2} \mathrm{Al}_{10}$ and $\mathrm{SmRu}_{2} \mathrm{Al}_{10}$ [46]. The $1 / \chi(T)$ plot of $\mathrm{SmFe}_{2} \mathrm{Al}_{10}$ showed a broad plateau-like region signifying the underlying valence fluctuation of the $\mathrm{Sm}^{3+}$ ions; however, such a feature is absent in the present Sm-compound. The large curvature of $\chi(T)$ in the case of $\mathrm{Sm}_{3} \mathrm{Ru}_{4} \mathrm{Ge}_{13}$ could hint at significant contribution from van Vleck term in the total susceptibility. Taking all this into consideration, and since a simple Curie-Weiss formula failed to fit the observed susceptibility, fits were performed to $\chi(T)$ employing a modified Curie-Weiss equation:

$$
\chi(T)=\frac{N_{A}}{k_{B}}\left[\frac{\mu_{\mathrm{eff}}^{2}}{3\left(T-\theta_{p}\right)}+\frac{20 \mu_{\mathrm{B}}^{2}}{7 E_{g}}\right]
$$

In the above equation, the first term corresponds to Curie-Weiss contribution from the $J=\frac{5}{2}$ ground state multiplet and the second term to the temperature independent van Vleck contribution from the excited $J=\frac{7}{2}$ multiplet. $E_{g}$ corresponds to the energy separation between the levels. However, a least-squares fit to the experimental $\chi(T)$ using Eqn (1) yielded only 0.12 (4) $\mu_{\mathrm{B}}$ for $\mu_{\mathrm{eff}}$ of $\mathrm{Sm}$ as the only magnetic species in $\mathrm{Sm}_{3} \mathrm{Ru}_{4} \mathrm{Ge}_{13}$. This is in contrast to the case of $\mathrm{SmRu}_{2} \mathrm{Al}_{10}$ where $\mu_{\mathrm{eff}}=0.85 \mu_{\mathrm{B}}$ was obtained hinting at more or less localized behaviour of $\mathrm{Sm}^{3+}$ moments. The reduction in the effective moment from the Russell-Saunders value of $0.84 \mu_{\mathrm{B}}$ in the present case could arise from crystalline electric field effects.

In Fig 5 (b), the magnetic susceptibility $\chi(T)$ of $\mathrm{Sm}_{3} \mathrm{Ru}_{4} \mathrm{Ge}_{13}$ for $T<25 K$ in three different applied fields $0.01 \mathrm{~T}$, $2 \mathrm{~T}$ and $4 \mathrm{~T}$ are plotted together. It can be noted that neither the position nor the shape of the peak at $T_{N}$ is altered with the application of magnetic field. Also, the curves are suggestive of the fact that above $2 \mathrm{~T}$, a certain insensitiveness to magnetic field sets in. The magnetic hysteresis at $2 \mathrm{~K}$ and $10 \mathrm{~K}$ are presented in Fig 5 (c). At $2 \mathrm{~K}$, a slight curvature is observed however at $10 \mathrm{~K}$, only a linear response is seen. A significantly reduced value of maximum magnetic moment is observed in $\mathrm{Sm}_{3} \mathrm{Ru}_{4} \mathrm{Ge}_{13}$ at $2 \mathrm{~K}, 7 \mathrm{~T}$. In Fig[5](d), a plot of field-cooled magnetization curves $\mathrm{Sm}_{3} \mathrm{Ru}_{4} \mathrm{Ge}_{13}$ in cooling and warming cycles of measurement applying 1000 Oe applied field are presented. It can be noticed that a weak yet, distinct thermal hysteresis is present around the $T_{N}$. The hysteresis opens wide in the proximity of $T_{N}$ and closes off at about $4.2 \mathrm{~K}$. It could be understood as a indication towards the presence of subtle structural modifications 
in $\mathrm{Sm}_{3} \mathrm{Ru}_{4} \mathrm{Ge}_{13}$ [40]. Moreover, a hint about close coupling between magnetic and lattice in $\mathrm{Sm}_{3} \mathrm{Ru}_{4} \mathrm{Ge}_{13}$ is also obtained from Fig 5 (d).

\subsection{Resistivity and magnetoresistance}

Figure 6 (a) illustrates the electrical resistivity, $\rho(T)$, in semi-log scale. A gradual increase of resistivity upon reduction of temperature is observed in $\mathrm{Sm}_{3} \mathrm{Ru}_{4} \mathrm{Ge}_{13}$. At around $5 \mathrm{~K}$, an abrupt dip is observed which corresponds to the magnetic phase transition at $T_{N}$ which was observed in magnetization as well as in specific heat (Fig 5 and Fig 4 respectively). The inset of (a) magnifies the data close to $5 \mathrm{~K}$. The overall nature of $\rho(T)$ does not support that of a good metal however the magnitude of resistivity is comparable to other quasi-skutterudites in this class like $\mathrm{Y}_{3} \mathrm{Ir}_{4} \mathrm{Ge}_{13}[25]$. An activated-type of resistivity behaviour was observed in many $R_{3} T_{4} X_{13}$ systems [47] hence, an analysis using $\rho=\rho_{0}[1+\exp (-\Delta / 2 T)]$ was attempted. However, the fit deviated from the data about $60 \mathrm{~K}$ and yielded a $\Delta$ value of $473.4(8) \mathrm{K}$. The $\Delta$ value is comparable to that obtained for $\mathrm{Y}_{3} \mathrm{Ir}_{4} \mathrm{Ge}_{13}$ [25] but is vastly different from systems like $\mathrm{CeOs}_{2} \mathrm{Al}_{10}[48]$. A curve-fit assuming $\rho \sim-\ln (\mathrm{T})$ pertinent to a Kondo system was attempted to find that the linear region extends over a significant range from $300 \mathrm{~K}$ to about $60 \mathrm{~K}$. However, magnetoresistance data (shown later) rules out the presence of Kondo effect. As a next step we analyzed the resistivity using a twoband model which describes that the prominent contribution to $\rho(T)$ arises from scattering of electrons within a broad conduction band and a narrow Lorentzian-shaped $4 f$ band. In such a scenario, the electronic density of states can be expressed as $N\left(\epsilon_{F}\right)=W /\left(W^{2}+P^{2}\right)$ where $W$ and $P$ represent the width and position of the $f$ band. Both $W$ and $P$ are temperature-dependent and are important in interpreting low temperature thermoelectric properties of Kondo systems. Within the two-band model, $\rho(T)$ is written as:

$$
\rho(T)=\rho_{0}+c T+D \frac{W}{W^{2}+P^{2}}
$$

where, $W=q_{f} \exp \left(-q_{f} / T\right)$ and $P=A+B \exp (-m / T) . A, B$ and $m$ are constants for a given compound; $q_{f}$ is the fluctuation temperature that provides a measure of the quasielastic linewidth governing the Abrikosov-Suhl resonance that arises from the hybridization between $4 f$ and conduction band. The residual resistivity is indicated as $\rho_{0}, c$ is phonon contribution and $D$ represents the strength of overlap of the $4 f$ band with the conduction band. In Fig 6 (b) a least-squares fit of the $\rho(T)$ data to Eqn (2) is shown. It can be seen that a satisfactory description of resistivity data is obtained using the two-band model. From the fit, $q_{f} \approx 25 \mathrm{~K}$ is obtained. A similar value of $30 \mathrm{~K}$ was obtained in the case of another caged structure compound, $\mathrm{CeOs}_{2} \mathrm{Al}_{10}$ [48]. The two-band model is not valid across the phase transition and hence fails to explain the region of $\rho(T)$ below about $40 \mathrm{~K}$.

The isothermal magnetoresistance, $\rho(\mathrm{H})$, of $\mathrm{Sm}_{3} \mathrm{Ru}_{4} \mathrm{Ge}_{13}$ at 2, 5 and $10 \mathrm{~K}$ are presented in (c). The $\rho(\mathrm{H})$ is observed to be positive. Interestingly, a nonlinear characteristic is displayed by $\rho(\mathrm{H})$ in the temperature region below the $T_{N}$ with a feature-rich low-field response and a gradual saturation up to $90 \mathrm{kOe}$. The $\rho(H)$ scans at $100 \mathrm{~K}$ and at $150 \mathrm{~K}$ show a $H$-dependence very similar to the metal-like features observed at $10 \mathrm{~K}$ ruling out the possibility of Kondo effect in this Sm quasi-skutterudite.

\subsection{Thermal conductivity}

The total thermal conductivity $\kappa_{T}(T)$, electrical resistivity $\rho(T)$ and thermopower $S(T)$ were measured simultaneously using the thermal transport option of the PPMS. The temperature dependence of total thermal conductivity, $\kappa_{T}(T)$, of $\mathrm{Sm}_{3} \mathrm{Ru}_{4} \mathrm{Ge}_{13}$ is presented in Fig 7 (a) as black open squares. The $\kappa_{T}(T)$ reduces by an order of magnitude while $\mathrm{Sm}_{3} \mathrm{Ru}_{4} \mathrm{Ge}_{13}$ is cooled from $300 \mathrm{~K}$ to $2 \mathrm{~K}$. The electronic contribution to the thermal conductivity, $\kappa_{e}(T)$ was extracted following the Wiedemann-Franz relation[49].

$$
\kappa_{e}(T)=\frac{L_{0} T}{\rho(T)}
$$

In this equation, the Lorenz number is $L_{0}=\left(\frac{\pi k_{B}^{2}}{e \sqrt{3}}\right)^{2}=2.54 \times 10^{-8} \mathrm{~W} \Omega K^{-2}$ and $\rho(T)$ is the electrical resistivity. In Fig $7(\mathrm{a}), \kappa_{e}(T)$ is represented as blue open circles. The phonon contribution $\kappa_{p h}(T)$ to the total thermal conductivity was estimated using the expression: $\kappa_{T}(T)=\kappa_{e}(T)+\kappa_{p h}(T)$. The $\kappa_{e}(T)$ estimated by Eqn (3p is about an order of magnitude lower than the total thermal conductivity. It is clear from Fig 7 that the thermal conductivity is dominated 
by phonons. The anomaly at $T_{N}$ which was observed in specific heat, magnetization and resistivity is clearly seen in thermal conductivity also. The region of anomaly is magnified in the inset of Fig 7 (a).

The total thermal conductivity, $\kappa_{T}(T)$ is plotted in a log-log scale in Fig 6 (b) where a relatively extended region of $T^{2}$-dependence is observed above the $T_{N}$. This feature supports the fact that the thermal conductivity is dominated by phononic contribution. In Fig 7 (c), a plot of normalized Lorenz number, $L / L_{0}$ is presented. Here, $L=\left(\frac{\kappa \rho}{T}\right)$ where $\kappa$ and $\rho$ are the measured thermal conductivity and resistivity respectively. The anomaly observed in $\kappa_{T}(T)$ at $T_{N}$ is enhanced in this plot and signifies that additional latent heat is involved in the magnetic phase transition. However, it must be noted that a thermal hysteresis was observed in magnetization (see Fig 5(d)) pointing towards the involvement of lattice degrees of freedom concurrent with the magnetic part. The experimentally measured thermopower, $S(T)$, is presented in the panel (d) of Fig 7. At $300 \mathrm{~K}, S(T)$ reaches a value of $39 \mu \mathrm{V} / \mathrm{K}$. This value may be compared with the values for pure metals, $1-10 \mu \mathrm{V} / \mathrm{K}$, and with that of semiconductors, $10^{2}-10^{3} \mu \mathrm{V} / \mathrm{K}$. The positive sign of the thermopower suggests that the conduction mechanism is hole dominated. The behavior of thermopower is qualitatively and quantitatively very similar to that of $\mathrm{Y}_{3} \mathrm{Ir}_{4} \mathrm{Ge}_{13}$, which too, displayed two distinct linear regions. The scenario described by the two-band model applies to the thermopower also since the Seebeck coefficient is proportional to the energy-derivative of the density of states at the Fermi level. However, a dome-shaped feature in thermopower is not observed in $\mathrm{Sm}_{3} \mathrm{Ru}_{4} \mathrm{Ge}_{13}$ which is normally observed in many compounds with intermediate valence states and CEF effects. In the inset of (d), a plot of thermopower is presented as $T / S$ versus $T^{2}$. A large region of linearity is observed in the high temperature region. The dash-dotted line is a linear fit to the high temperature region above $200 \mathrm{~K}$. Within the Boltzmann theory and relaxation time approximation for an isotropic system, the thermopower can be approximated as $S=A T /\left[B^{2}+T^{2}\right]$ where $A=\frac{2\left(\epsilon_{0}-\epsilon_{F}\right)}{|e|}$ and $B^{2}=\left(3 / \pi^{2} k_{B}^{2}\right)\left(\left(\epsilon_{0}-\epsilon_{F}\right)^{2}+\Gamma^{2}\right)[50]$. From the plot of $T / S$ versus $T^{2}$, the constants $A$ and $B$ can be determined. This leads to an estimate of $\left(\epsilon_{0}-\epsilon_{F}\right)=$ $0.07 \mathrm{meV}$ and $\Gamma=0.12 \mathrm{meV}$.

\section{Discussion}

The present set of data on the magnetic, thermodynamic and transport properties of the quasi-skutterudite, $\mathrm{Sm}_{3} \mathrm{Ru}_{4} \mathrm{Ge}_{13}$, reveal a magnetic transition at $T_{N} \approx 5 \mathrm{~K}$ implying strong correlation between all the measured physical properties. The caged structure of the present composition is known to be conducive for atomic "rattling" phenomena known in this class of materials [25, 26]. From the structural analysis of $\mathrm{x}$ ray data, it is concluded that the crystal structure conforms to the cubic space group $P m \overline{3} n$ which is commonly found in $R_{3} T_{4} X_{13}$ compounds. However, indications of subtle structural distortions inherent to this type of materials are present in the $\mathrm{x}$ ray data [22, 27, 40]. The lattice parameters of $\mathrm{Sm}_{3} \mathrm{Ru}_{4} \mathrm{Ge}_{13}$ estimated from the $\mathrm{x}$ ray data is plotted along with the lattice parameters for $R_{3} \mathrm{Ru}_{4} \mathrm{Ge}_{13}$ taken from Ref.[47] in panel (c) of Fig 3. No sign of a structural anomaly is observed for the Sm compound from such a plot. However, the superstructure-like peaks observed in the $\mathrm{x}$ ray pattern is suggestive of subtle structural distortions that may be present in $\mathrm{Sm}_{3} \mathrm{Ru}_{4} \mathrm{Ge}_{13}$.

The magnetic susceptibility of $\mathrm{Sm}_{3} \mathrm{Ru}_{4} \mathrm{Ge}_{13}$ shows indications of crystal electric field effects arising from the close-lying crystal field levels of $\mathrm{Sm}^{3+}$ ions. The $\chi(T)$ behavior is qualitatively different from other Sm-based systems like $\mathrm{SmFe}_{2} \mathrm{Al}_{10}$ where strong valence fluctuations have been reported[46]. However a simple Curie-Weiss description was not suitable for $\mathrm{Sm}_{3} \mathrm{Ru}_{4} \mathrm{Ge}_{13}$ and van Vleck terms in the total magnetic susceptibility were required to fit the data. The typical response of $\chi(T)$ of compounds that displays valence fluctuation show a profound minimum which is due to the instability of $4 f$ shell and strong hybridization between the $4 f$ electron and conduction electron states[51]. The magnetic susceptibility of $\mathrm{Sm}_{3} \mathrm{Ru}_{4} \mathrm{Ge}_{13}$ show a very weak variation of magnitude starting from $300 \mathrm{~K}$ toward $2 \mathrm{~K}$. These features rule out the possibility of valence fluctuation though, mixed valence of Sm may be present. However, the field-insensitive features in specific heat and the very low magnetic moment values attained in magnetization measurements at low temperature point toward the possibility of octupolar ordering mechanisms operating in this Sm-based 3:4:13 alloy similar to the case of filled skutterudites or Frank-Kasper cages.

The specific heat of $\mathrm{Sm}_{3} \mathrm{Ru}_{4} \mathrm{Ge}_{13}$ displays strong robustness of the phase transition at $T_{N} \approx 5 \mathrm{~K}$ in applied magnetic fields up to $5 \mathrm{~T}$. This feature is very similar to the observation in other $\mathrm{Sm}$-based caged-structures, $\mathrm{SmTa}_{2} \mathrm{Al}_{20}$ and $\mathrm{SmTi}_{2} \mathrm{Al}_{20}[13,16]$. A curve-fit to the total observed specific heat could be advanced through a model description incorporating a Debye term, three Einstein terms and a linear term in specific heat. The magnetic entropy estimated by subtracting the phonon and electronic part from the total specific heat indicates the crystal field effects of Sm. Though 
the Einstein terms were required to describe the phononic part, no indication of "rattling" is obtained from the plot of $\left(C_{p}-\gamma T\right) / T^{3}$ versus $T$. Moreover, a simple description of specific heat following $C_{p}(T) \sim T^{2}$ does not hold in the ordered region below the $T_{N}$ suggesting that $\mathrm{Sm}_{3} \mathrm{Ru}_{4} \mathrm{Ge}_{13}$ may not be a simple antiferromagnet. The low temperature estimate of Sommerfeld coefficient suggests the formation of heavy quasi particles. A comparison of Sommerfeld coefficients and Debye temperatures of various Ge-based 3:4:13 alloys is presented in Table 2

Though the electrical resistivity of $\mathrm{Sm}_{3} \mathrm{Ru}_{4} \mathrm{Ge}_{13}$ exhibited a $\ln T$ behaviour in a wide temperature above $\approx 60 \mathrm{~K}$ indicating possible Kondo physics, the field-dependence of resistivity, $\rho(H)$, at different temperatures rules out such a scenario. At temperatures below $T_{N}$, the $\rho(H)$ is nonlinear and shows an initial increase in low fields which, then decreases as the applied field is increased. However, above $10 \mathrm{~K}$, the $\rho(H)$ shows a monotonic decrease with applied field which is more metal-like. Even at $100 \mathrm{~K}$ and $150 \mathrm{~K}$, where the $\ln T$ behavior was observed, a monotonic variation of $\rho(H)$ was observed (data not presented). Interestingly, the field-insensitive features observed in specific heat was also reflected in the field-dependence of the Sommerfeld coefficient, $\gamma$, and the coefficient $A$. An unusual fieldinsensitiveness of $\gamma$ and $A$ up to $5 \mathrm{~T}$ was clear from Fig 6(d). Similar to many other Sm-based caged systems, this field-insensitiveness might have its origin in octupolar ordering [17, 16]. The phase transition observed in magnetization, specific heat and resistivity at $T_{N} \approx 5 \mathrm{~K}$ is also present in the thermal conductivity data. This might serve as an indication for the coupling between all degrees of freedom in this material.

The Sm-containing filled skutterudites $\mathrm{Sm}_{4} X_{12}(T=$ transition metal, $X=\mathrm{Ge}, \mathrm{P}, \mathrm{Sb})$ are compounds that can be readily compared with the present $\mathrm{Sm}$-based quasi skutterudite. $\mathrm{SmFe}_{4} \mathrm{P}_{12}$ show Kondo behaviour[52] as evidenced through the relaxation mechanisms through ${ }^{31} \mathrm{P}-\mathrm{NMR}$ and $\mu \mathrm{SR}$ experiments [53]. The crystalline fields of $\mathrm{Sm}^{3+}$ showed their presence as a ground state and an excited state at $70 \mathrm{~K}$ and a ferromagnetic ground state was inferred to exist below 1.6 K. Another experimental investigation of the same compound using de Haas-van Alphen effect, magnetoresistance and Hall effect revealed anomalous large anisotropy in cyclotron effective mass despite a near-spherical Fermi surface[54]. In this Sm-compound also, the mass enhancement was found to be robust against applied magnetic field. In a specific heat study combining the filled skutterudites $\mathrm{SmFe}_{4} \mathrm{P}_{12}, \mathrm{SmRu}_{4} \mathrm{P}_{12}$ and $\mathrm{SmOs}_{4} \mathrm{P}_{12}$, signatures of orbital and antiferromagnetic orderings were obtained for the Ru-based compound[18]. The Pt-based filled skutterudite $\mathrm{SmPt}_{4} \mathrm{Ge}_{12}$ has $\mathrm{Sm}$ in a temperature-independent intermediate valence state while signatures of heavy fermion and Kondo effects are seen[55]. These reports indicate that Sm-containing skutterudites and quasi-skutterudites are interesting compounds where anomalous behaviour originating from rare earth magnetism, anisotropy and dipolar interactions are observed.

\section{Conclusions}

Strong field-insensitive specific heat and heavy fermion features are observed in the quasi-skutterudite $\mathrm{Sm}_{3} \mathrm{Ru}_{4} \mathrm{Ge}_{13}$ The unusual field-insensitiveness is also present in $\gamma$ as well. Very low values of effective magnetic moment and maximum moment in field-scans of magnetization point toward possible octupolar ordering mechanisms being operative. The magnetoresistance below the $T_{N}$ display nonlinear characteristics but behave metal-like above the $T_{N}$. The features observed in the present Sm based 3:4:13 alloys bear strong resemblances to the unusual heavy fermion and field-insensitive features observed in Sm-based filled skutterudites and Frank-Kasper cages.

\section{Acknowledgements}

HSN and RKK acknowledge FRC/URC for a postdoctoral fellowship. AMS thanks the SA-NRF (93549) and the FRC/URC of UJ for financial assistance.

\section{References}

[1] C. Uher, Semicond. Semimet. 69 (2001) 139-253.

[2] B. C. Sales, Handbook on the Physics and Chemistry of Rare Earths 33 (2003) 1, ed. Gschneidner, K. A. Jr. and Bünzli, J.-C. G. and Pecharsky, V. K., Elsevier, Amsterdam.

[3] G. S. Nolas, D. T. Morelli, T. M. Tritt, Annu. Rev. Mater. Sci. 29 (1) (1999) 89-116.

[4] A. Leithe-Jasper, W. Schnelle, H. Rosner, N. Senthilkumaran, A. Rabis, M. Baenitz, A. Gippius, E. Morozova, J. A. Mydosh, Y. Grin, Phys. Rev. Lett. 91 (3) (2003) 037208. 
[5] K. Matsuhira, M. Wakeshima, Y. Hinatsu, C. Sekine, I. Shirotani, D. Kikuchi, H. Sugawara, H. Sato, J. Magn. Magn. Mater. 310 (2) (2007) $226-228$.

[6] N. Ogita, R. Kojima, Y. Takasu, T. Hasegawa, T. Kondo, M. Udagawa, N. Takeda, T. Ikeno, K. Ishikawa, H. Sugawara, et al., J. Magn. Magn. Mater. 310 (2) (2007) 948-950.

[7] S. Sanada, Y. Aoki, H. Aoki, A. Tsuchiya, D. Kikuchi, H. Sugawara, H. Sato, J. Phys. Soc. Jpn. 74 (1) (2005) $246-249$.

[8] W. M. Yuhasz, N. A. Frederick, P. C. Ho, N. P. Butch, B. J. Taylor, T. A. Sayles, M. B. Maple, J. B. Betts, A. H. Lacerda, P. Rogl, et al., Phys. Rev. B 71 (10) (2005) 104402.

[9] M. Mizumaki, S. Tsutsui, H. Tanida, T. Uruga, D. Kikuchi, H. Sugawara, H. Sato, J. Phys. Soc. Jpn. 76 (5).

[10] A. Yamasaki, S. Imada, H. Higashimichi, H. Fujiwara, T. Saita, T. Miyamachi, A. Sekiyama, H. Sugawara, D. Kikuchi, H. Sato, et al., Phys. Rev. Lett. 98 (15) (2007) 156402.

[11] H. Sato, H. Sugawara, Y. Aoki, H. Harima, Handbook of Magnetic Materials, vol. 18, North-Holland, Amsterdam, 2009.

[12] E. D. Bauer, N. A. Frederick, P. C. Ho, V. S. Zapf, M. B. Maple, Phys. Rev. B 65 (10) (2002) 100506.

[13] A. Yamada, R. Higashinaka, R. Miyazaki, K. Fushiya, T. D. Matsuda, Y. Aoki, W. Fujita, H. Harima, H. Sato, J. Phys. Soc. Jpn. 82 (12).

[14] Y. Isikawa, T. Mizushima, J. Ejiri, T. Kuwai, J. Phys. Soc. Jpn. 83 (7).

[15] A. Sakai, S. Nakatsuji, J. Phys. Soc. Jpn. 81 (Suppl. B).

[16] R. Higashinaka, T. Maruyama, A. Nakama, R. Miyazaki, Y. Aoki, H. Sato, J. Phys. Soc. Jpn. 80 (9) (2011) 093703.

[17] Y. Aoki, S. Sanada, D. Kikuchi, H. Sugawara, H. Sato, J. Phys. Soc. Jpn. 76 (11) (2007) 113703.

[18] K. Matsuhira, Y. Doi, M. Wakeshima, Y. Hinatsu, H. Amitsuka, Y. Shimaya, R. Giri, C. Sekine, I. Shirotani, J. Phys. Soc. Jpn. 74 (3) (2005) $1030-1035$

[19] N. Takeda, M. Ishikawa, Physica B 329 (2003) 460-461.

[20] J. P. Remeika, G. P. Espinosa, A. S. Cooper, H. Barz, J. M. Rowell, D. B. McWhan, J. M. Vandenberg, D. E. Moncton, Z. Fisk, L. D. Woolf, et al., Solid State Commun. 34 (12) (1980) 923-926.

[21] J. M. Vandenberg, Mater. Res. Bull. 15 (6) (1980) 835-847.

[22] J. L. Hodeau, J. Chenavas, M. Marezio, J. P. Remeika, Solid State Commun. 36 (10) (1980) 839-845.

[23] N. Kase, H. Hayamizu, K. Inoue, J. Akimitsu, Physica C 471 (21) (2011) 711-713.

[24] P. D. Kulkarni, S. S. Banerjee, C. V. Tomy, G. Balakrishnan, D. M. Paul, S. Ramakrishnan, A. K. Grover, Phys. Rev. B 84 (1) (2011) 014501.

[25] A. M. Strydom, J. Phys.: Condens. Matter 19 (38) (2007) 386205.

[26] A. M. Strydom, N. Oeschler, F. Steglich, Physica B 403 (5) (2008) 746-748.

[27] J. L. Hodeau, M. Marezio, J. P. Remeika, C. H. Chen, Solid State Commun. 42 (2) (1982) 97-102.

[28] B. Eisenmann, H. Schäfer, J. Less Comm. Metals 123 (1986) 89-94.

[29] C. U. Segre, H. F. Braun, K. Yvon, Ternary Superconductors (1981) 243-246Ed. G. K. Shenoy.

[30] V. A. Bruskov, V. K. Pecharskij, O. I. Bodak, Izv. Akad. Nauk. SSSR Neorg. Mater. 22 (1986) 1471.

[31] Y. Mudryk, A. Grytsiv, P. Rogl, C. Dusek, A. Galatanu, E. Idl, H. Michor, E. Bauer, C. Godart, D. Kaczorowski, et al., J. Phys.: Condens. Matter 13 (33) (2001) 7391.

[32] D. Niepmann, R. Pöttgen, K. M. Poduska, F. J. DiSalvo, H. Trill, B. D. Mosel, Z Natur. 56b (2001) 1-8.

[33] C. Nagoshi, R. Yamamoto, K. Kuwahara, H. Sagayama, D. Kawana, M. Kohgi, H. Sugawara, Y. Aoki, H. Sato, T. Yokoo, et al., J. Phys. Soc. Jpn. 75 (4).

[34] P. Bordet, D. E. Cox, G. P. Espinosa, J. L. Hodeau, M. Marezio, Solid State Commun. 78 (5) (1991) $359-366$.

[35] H. M. Rietveld, J. Appl. Cryst. 2 (1969) 65.

[36] J. Rodriguez-Carvajal, Recent advances in magnetic structure determination by neutron powder diffraction, Physica B 192 (1993) 55

[37] E. Jarosewich, L. A. Boatner, Geostandards Newsletter 15 (2) (1991) 397.

[38] J. J. Donovan, J. M. Hanchar, P. M. Picolli, M. D. Schrier, L. A. Boatner, E. Jarosewich, J. Res. Natl. Inst. Stand. Technol. 107 (6) (2002) 693.

[39] C. Merlet, Mikrochim.Acta 114/115 (1994) 363.

[40] R. Gumeniuk, L. Akselrud, K. O. Kvashnina, W. Schnelle, A. A. Tsirlin, C. Curfs, H. Rosner, M. Schöneich, U. Burkhardt, U. Schwarz, et al., Dalton Trans. 41 (20) (2012) 6299-6309.

[41] C. P. Susz, J. Muller, K. Yvon, E. Parthé, J. Less Comm. Metals 71 (1) (1980) P1-P8.

[42] M. A. Hayward, R. J. Cava, J. Phys.: Condens. Matter 14 (25) (2002) 6543.

[43] C. L. Yang, X. Wang, X. Zhang, D. S. Wu, M. Liu, P. Zheng, J. Y. Yao, Z. Z. Li, Y. F. Yang, Y. G. Shi, et al., Phys. Rev. B 91 (7) (2015) 075120.

[44] A. M. Strydom, Acta Phys. Pol. A 126 (1) (2014) 318-321.

[45] H. W. De Wijn, A. M. Van Diepen, K. H. J. Buschow, Phys. Rev. 161 (2) (1967) 253.

[46] P. Peratheepan, A. M. Strydom, J. Phys.: Condens. Matter 27.

[47] K. Ghosh, S. Ramakrishnan, G. Chandra, Phys. Rev. B 48 (14) (1993) 10435.

[48] C. S. Lue, H. F. Liu, B. D. Ingale, J. N. Li, Y. K. Kuo, Phys. Rev. B 85 (24) (2012) 245116.

[49] C. Kittel, Introduction to Solid State Physics, Wiley, New York, 1996.

[50] U. Gottwick, K. Gloss, S. Horn, F. Steglich, N. Grewe, J. Magn. Magn. Mater. 47 (1985) 536-538

[51] M. Falkowski, A. M. Strydom, J. Phys.: Condens. Matter 27 (39) (2015) 395601.

[52] N. Takeda, H. Mitamura, K. Kindo, J. Phys. Soc. Jpn. 77 (Suppl. A) (2008) 209-210

[53] K. Hachitani, H. Fukazawa, Y. Kohori, I. Watanabe, Y. Yoshimitsu, K. Kumagai, R. Giri, C. Sekine, I. Shirotani, J. Phys. Soc. Jpn. 75 (12) (2006) 124717.

[54] D. Kikuchi, H. Sugawara, K. Tanaka, H. Aoki, M. Kobayashi, S. Sanada, K. Kuwahara, Y. Aoki, H. Shishido, R. Settai, et al., J. Phys. Soc. Jpn. 77 (11) (2008) 114705.

[55] R. Gumeniuk, M. Schöneich, A. Leithe-Jasper, W. Schnelle, M. Nicklas, H. Rosner, A. Ormeci, U. Burkhardt, M. Schmidt, U. Schwarz, et al., New J. Phys. 12 (10) (2010) 103035. 
[56] K. Ghosh, S. Ramakrishnan, S. K. Dhar, S. K. Malik, G. Chandra, V. K. Pecharsky, K. A. Gschneidner Jr, Z. Hu, W. B. Yelon, Phys. Rev. B 52 (10) (1995) 7267.

[57] K. Momma, F. Izumi, J. Appl. Cryst. 41 (3) (2008) 653-658. 
Table 1: The atomic positions in the $P m \overline{3} n$ model used to refine the $\mathrm{x}$ ray data of $\mathrm{Sm}_{3} \mathrm{Ru}_{4} \mathrm{Ge}_{13}$. The refined lattice parameter is $a(\AA)=9.0171(2)$. The reliability factors of the fit are $\chi^{2}=4.5, R_{p}=6.1$ and $R_{w p}=8.8$. Wyck. stands for Wyckoff position and $B_{\text {iso }}$ are the isotropic thermal parameters taken from [43]. The occupancies were fixed to nominal values.

\begin{tabular}{llllll}
\hline \hline Atom & Wyck. & $x$ & $y$ & $z$ & $B_{\text {iso }}$ \\
\hline \hline $\mathrm{Sm}$ & $6 c$ & 0.25 & 0 & 0.5 & 0.001 \\
$\mathrm{Ru}$ & $8 e$ & 0.25 & 0.25 & 0.25 & 0.001 \\
$\mathrm{Ge}(1)$ & $2 a$ & 0 & 0 & 0 & 0.001 \\
$\mathrm{Ge}(2)$ & $24 k$ & 0 & 0.1519 & 0.3149 & 0.010 \\
\hline \hline Binary & & S.G. & $a$ & \multicolumn{3}{c}{$b$} & \\
\hline \hline $\mathrm{Ru}_{2} \mathrm{Ge}_{3}(\mathrm{o})$ & Pbcn & 11.4349 & 9.2423 & 5.7200 \\
\hline
\end{tabular}


Table 2: The Sommerfeld coefficients $(\gamma)$ and Debye temperatures $\left(\Theta_{D}\right)$ of a collection of Ge-based $R_{3} T_{4} X_{13}$ quasi-skutterudites. The abbreviations are M: metal, HF: heavy fermion, SC: superconductor, sm: semi-metal, K: Kondo system.

\begin{tabular}{lllll}
\hline \hline$R_{3} T_{4} \mathrm{Ge}_{13}$ & Type & $\Theta_{D}$ & Ref. \\
\hline \hline $\mathrm{Ce}_{3} \mathrm{Ru}_{4} \mathrm{Ge}_{13}$ & $\mathrm{HF}$ & $111 \mathrm{~mJ} / \mathrm{mol} \mathrm{K}^{2}$ & $187 \mathrm{~K}$ & {$[56]$} \\
$\mathrm{Yb}_{3} \mathrm{Co}_{4.3} \mathrm{Ge}_{12.7}$ & $\mathrm{SC}$ & $2.3 \mathrm{~mJ} / \mathrm{mol} \mathrm{K}^{2}$ & $207 \mathrm{~K}$ & {$[31]$} \\
$\mathrm{Yb}_{3} \mathrm{Pt}_{4} \mathrm{Ge}_{13}$ & & $<50 \mathrm{~mJ} / \mathrm{mol} \mathrm{K}^{2}$ & $310 \mathrm{~K}$ & {$[40]$} \\
$\mathrm{Yb}_{3} \mathrm{Rh}_{4} \mathrm{Ge}_{13}$ & & $25 \mathrm{~mJ} / \mathrm{mol} \mathrm{Y} \mathrm{K}^{2}$ & - & {$[44]$} \\
$\mathrm{Yb}_{3} \mathrm{Os}_{4} \mathrm{Ge}_{13}$ & $\mathrm{~K}$ & $14 \mathrm{~mJ} / \mathrm{mol} \mathrm{Y} \mathrm{K}$ & $160 \mathrm{~K}$ & {$[43]$} \\
$\mathrm{Y}_{3} \mathrm{Co}_{4} \mathrm{Ge}_{13}$ & $\mathrm{M}$ & $5.7 \mathrm{~mJ} / \mathrm{mol} \mathrm{Y} \mathrm{K}$ & $354 \mathrm{~K}$ & {$[56]$} \\
$\mathrm{Y}_{3} \mathrm{Ir}_{4} \mathrm{Ge}_{13}$ & $\mathrm{sm}$ & $4.3 \mathrm{~mJ} / \mathrm{mol} \mathrm{K}^{2}$ & $129 \mathrm{~K}$ & {$[25]$} \\
$\mathrm{Lu}_{3} \mathrm{Rh}_{4} \mathrm{Ge}_{13}$ & & $5 \mathrm{~mJ} / \mathrm{mol} \mathrm{Y} \mathrm{K}$ & - & {$[44]$} \\
$\mathrm{Sm}_{3} \mathrm{Ru}_{4} \mathrm{Ge}_{13}$ & $\mathrm{HF}, \mathrm{K}$ & $220 \mathrm{~mJ} / \mathrm{mol} \mathrm{Sm} \mathrm{K}^{2}$ & $280 \mathrm{~K}$ & {$[$ This work] } \\
\hline \hline
\end{tabular}




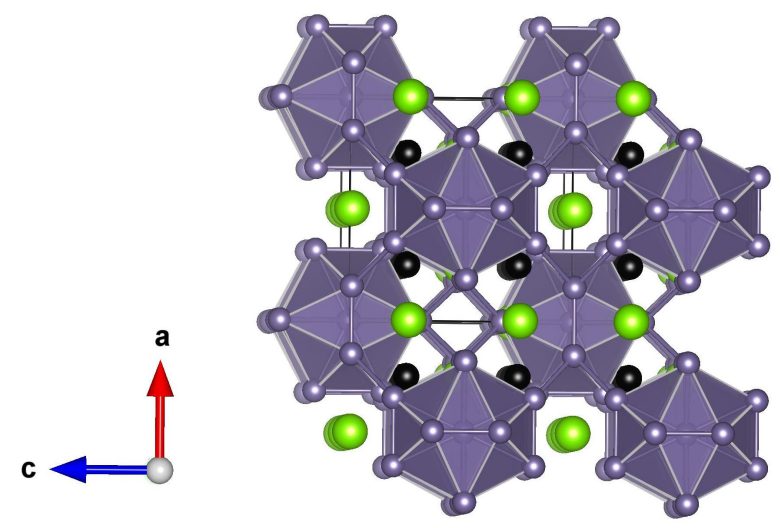

Figure 1: (color online) The structural motif of $\mathrm{Sm}_{3} \mathrm{Ru}_{4} \mathrm{Ge}_{13}$ showing the Ge-polyhedra forming the caged structure. The green spheres are $\mathrm{Sm}$, the black are Ru and the grey are Ge. The figure was prepared using VESTA[57]. 


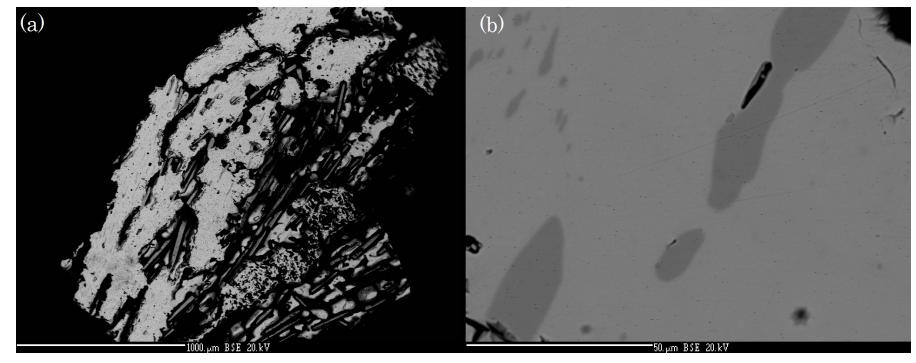

Figure 2: (color online) The metallographic image obtained from the surface of $\mathrm{Sm}_{3} \mathrm{Ru}_{4} \mathrm{Ge}_{13}$ through electron microprobe analysis. Different magnifications are shown in (a) and in (b). The darker regions in (b) correspond to $\mathrm{Ru}_{2} \mathrm{Ge}_{3}$ binary phase. 

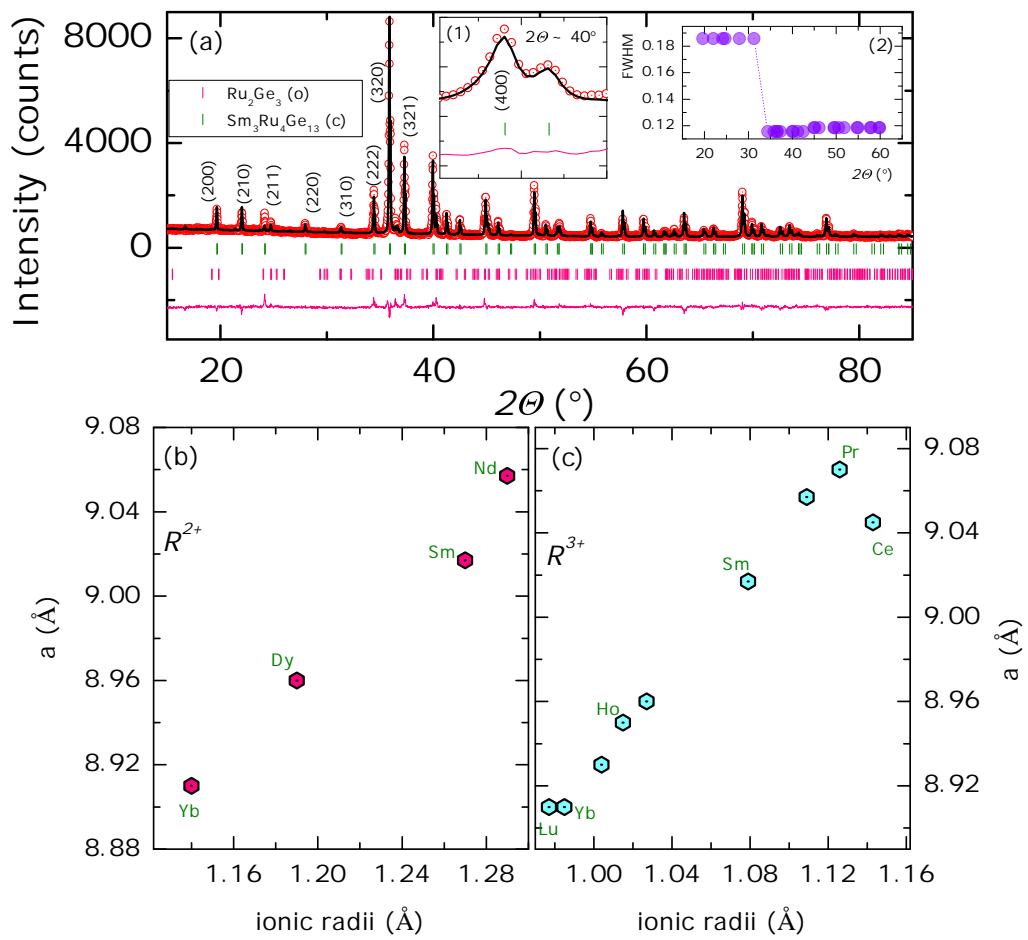

Figure 3: (color online) (a) The $\mathrm{x}$ ray diffraction pattern of $\mathrm{Sm}_{3} \mathrm{Ru}_{4} \mathrm{Ge}_{13}$ (red circles). The black solid line is the calculated pattern assuming $P m \overline{3} n$ space group for $\mathrm{Sm}_{3} \mathrm{Ru}_{4} \mathrm{Ge}_{13}$ and the binary phase, $\mathrm{Ru}_{2} \mathrm{Ge}_{3}$ (orthorhombic (o)). The vertical tick-marks are the allowed Bragg positions and the difference pattern is shown as a solid line. The inset (1) of (a) shows enlarged region around $40^{\circ}$ and (2) presents a plot of FWHM vs $2 \Theta$. Plots showing the lattice parameter variation of the $R_{3} \mathrm{RuGe}_{13}$ as a function of ionic radius of (b) $R^{2+}$ and (c) $R^{3+}$ with Sm-values incorporated from the present work. The data for other $R$ 's are obtained from Ref.[47]. 

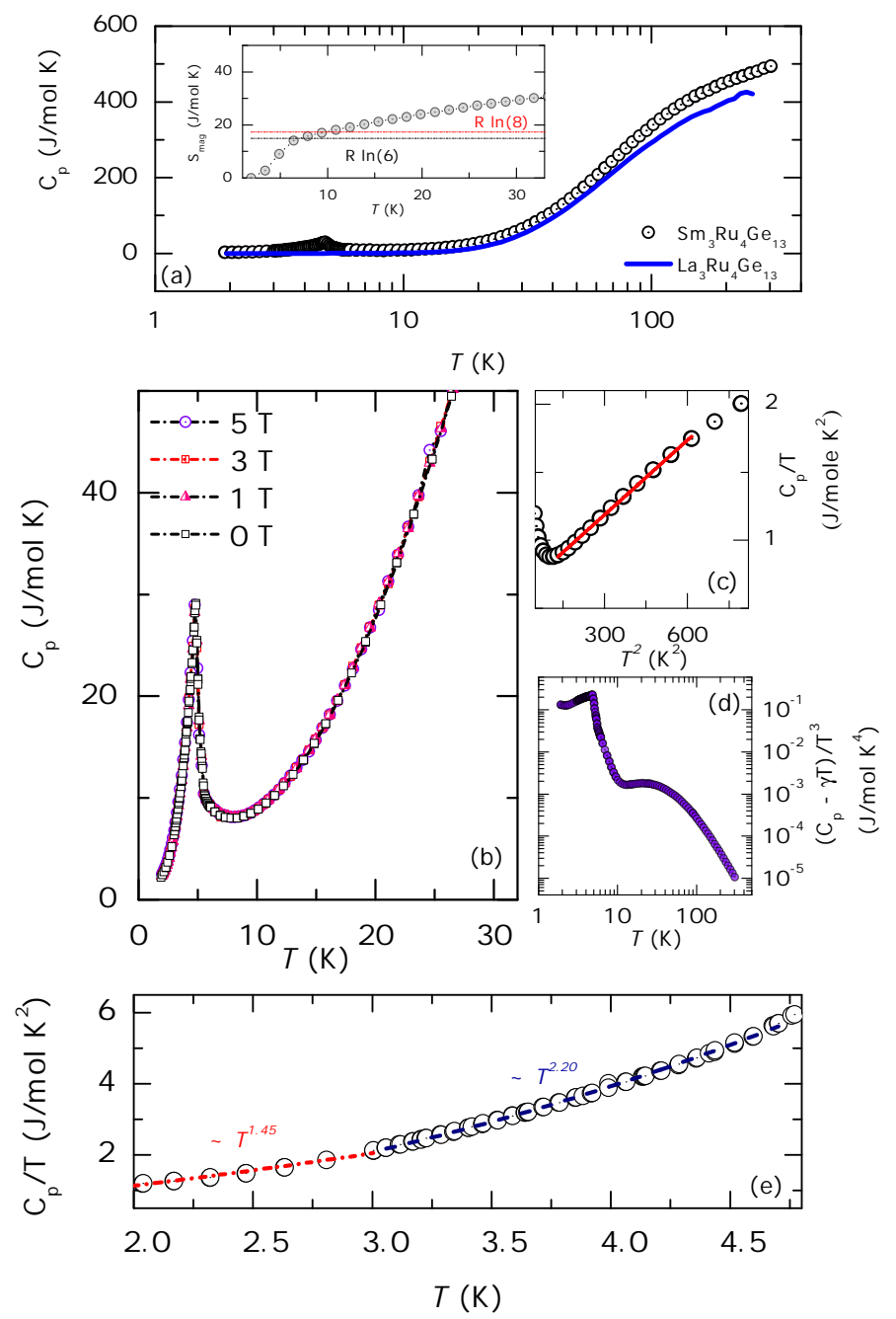

Figure 4: (color online) The specific heat $C_{p}$ of $\mathrm{Sm}_{3} \mathrm{Ru}_{4} \mathrm{Ge}_{13}$ is presented in (a) as black open circles and that of the non-magnetic La $\mathrm{Ru}_{4} \mathrm{Ge}_{13}$ as a blue solid line. The inset displays the magnetic entropy, $S_{\text {mag }}(\mathrm{b})$ Field-dependent specific heat reveals the robustness of $C_{p}(T)$ up to 5 T. (c) Shows a plot of $C_{p} / T$ versus $T^{2}$ used for a linear fit (solid line) which yielded $\gamma \approx 220 \mathrm{~mJ} / \mathrm{mol}_{-}-\mathrm{Sm} \mathrm{K}^{2}$. (d) Shows a log-log plot of $\left(C_{p}(T)-\gamma T\right) / T^{3}$ versus $T$ which shows only a weak and broad feature from phonon modes centered about $30 \mathrm{~K}$. (e) $C_{p}(T) / T$ below $T_{N}$ with two regions displaying different temperature-dependencies viz., $T^{1.45}$ and $T^{2.20}$. 

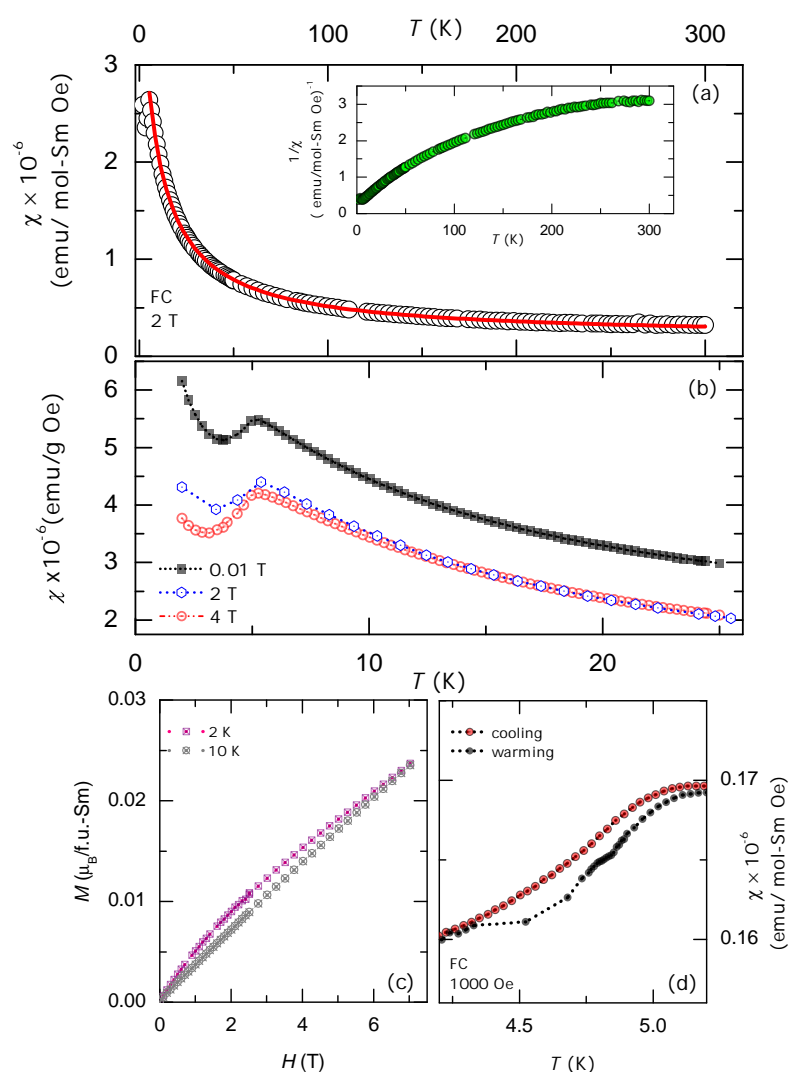

Figure 5: (color online) The magnetic response of $\mathrm{Sm}_{3} \mathrm{Ru}_{4} \mathrm{Ge}_{13}$. (a) Shows the field-cooled magnetization as a function of temperature measured at $2 \mathrm{~T}$ applied field. An anomaly at $T_{N} \approx 5 \mathrm{~K}$ is observed. The solid red line in (a) is a fit assuming modified Curie-Weiss law as described in Eqn (1). The inset shows the non-linear nature of $1 / \chi(T)$. (b) The $\chi(T)$ plots are various applied fields show insensitive features for higher fields. (c) Shows the field-scans of magnetization at $2 \mathrm{~K}$ and $10 \mathrm{~K}$. (d) Shows the presence of a weak but clear thermal hysteresis around $T_{N}$ measured in field-cooling protocol in $0.1 \mathrm{~T}$. 

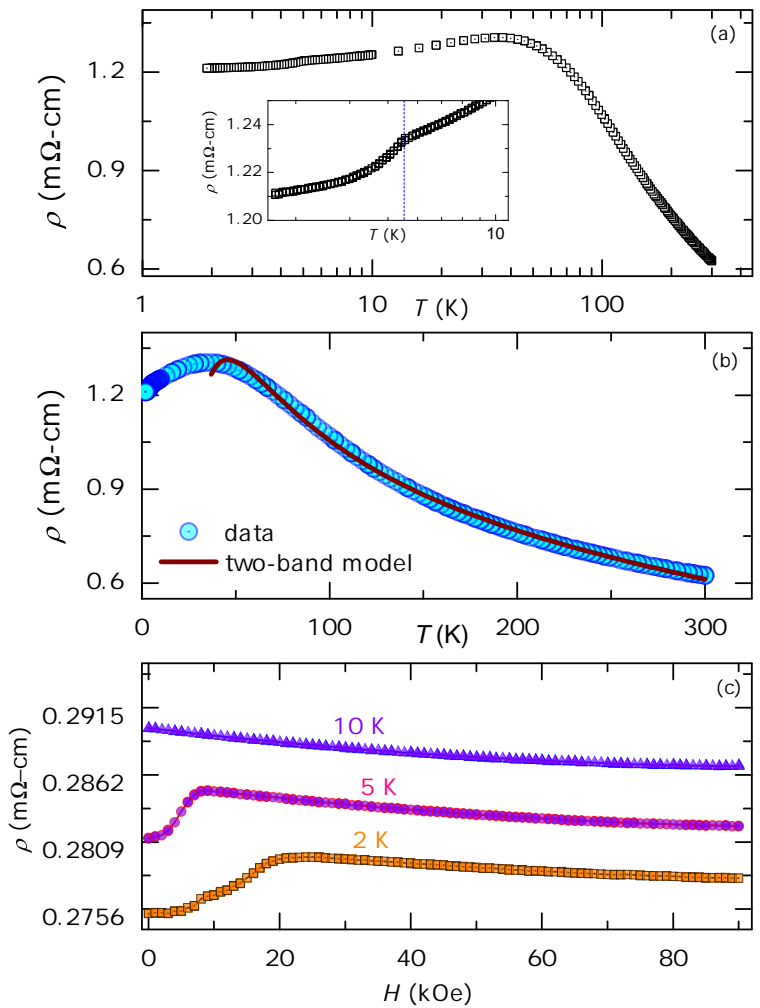

Figure 6: (color online) The electrical transport and magnetoresistance of $\mathrm{Sm}_{3} \mathrm{Ru}_{4} \mathrm{Ge}_{13}$. (a) Shows the electrical resistivity as a function of temperature $\rho(T)$ plotted in semi-log axes. The phase transition at $T_{N} \approx 5 \mathrm{~K}$ is magnified in the inset and highlighted using a vertical line. (b) Displays the fit to $\rho(T)$ employing the two-band model as described in Eqn (2). (c) The isothermal magnetoresistance of $\mathrm{Sm}_{3} \mathrm{Ru}_{4} \mathrm{Ge}_{13}$ at 2, 5 and $10 \mathrm{~K}$. (d) The magnetic field dependence of $\gamma$ and $A$. 

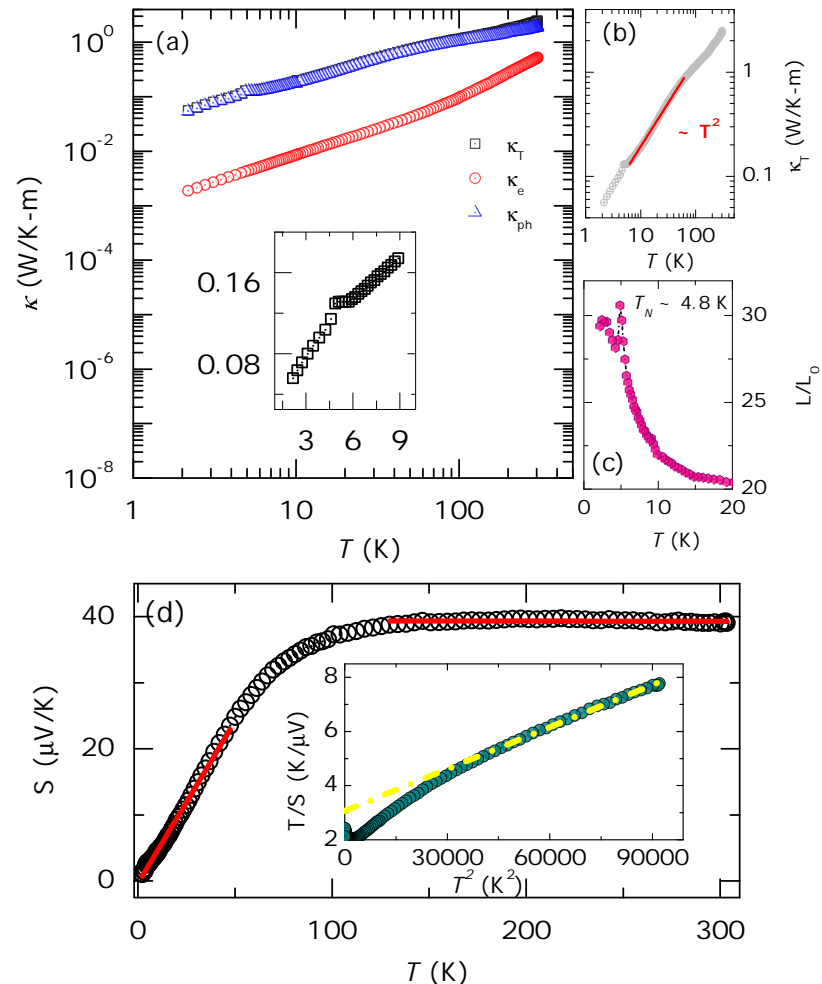

Figure 7: (color online) (a) The total thermal conductivity, $\kappa_{T}(T)$, of $\mathrm{Sm}_{3} \mathrm{Ru}_{4} \mathrm{Ge}_{13}$ plotted along with the electronic contribution, $\kappa_{e}(T)$ and the phonon contribution, $\kappa_{p h}(T)$. The inset shows the magnified region around $5 \mathrm{~K}$ where an anomaly is identified which matches with that found in the magnetization and specific heat. (b) Shows a log-log plot of $\kappa_{T}(T)$ which exhibits $\sim T^{2}$ dependence in the region above the transition. (c) The ratio, $L / L_{0}$ reflecting the anomaly at $T_{N}$. (d) Shows the Seebeck coefficient, $S$, with a fairly large value of $\approx 40 \mu \mathrm{V} / \mathrm{K}$ at $300 \mathrm{~K}$. The inset shows the plot of $T / S$ versus $T^{2}$ with a linear fit shown in dash-dotted line. 